\title{
Effect of Surfactant on Magnetic and Optical Properties of $\alpha-\mathrm{Fe}_{2} \mathrm{O}_{3}$ Nanoparticles
}

\author{
I.M. Mirza*, A.K. Sarfraz And S.K. Hasanain \\ Department of Physics, Quaid-i-Azam University, Islamabad, Pakistan \\ (Received January 26, 2014; in final form November 6, 2014)
}

\begin{abstract}
Hematite $\left(\alpha-\mathrm{Fe}_{2} \mathrm{O}_{3}\right)$ nanoparticles and hematite nanoparticles coated with polyvinylpyrrolidone (PVP) are synthesized chemically by co-precipitation. Prolysis and microemulsion methods respectively. An average size of nanoparticles (both coated and uncoated) was found by the broadening of the X-ray diffraction peaks using Scherrer's formula. It was found that coating reduced particle sizes. The attachment of the polymer on the surface of particles was confirmed by the Fourier transform infrared spectroscopy and thermogravimetric analysis. Optical ultraviolet/visible reflectance test also indicated the presence of PVP in coated nanohematites. In magnetic studies, it was observed that coating does not change main magnetic character of the $\alpha-\mathrm{Fe}_{2} \mathrm{O}_{3}$ nanoparticles however it reduces DC magnetization (as a function of applied field and temperature) to a considerable amount. Moreover it was observed that after coating Morin transition temperature $T_{\mathrm{M}}$ and its width $\Delta T_{\mathrm{M}}$ shifts to lower values, which is another indication of size reduction.
\end{abstract}

DOI: $10.12693 /$ APhysPolA.126.1280

PACS: 78.66.Qn, 75.47.Lx, 78.67.Bf, 75.75.-c, 78.67.-n

\section{Introduction}

Over the years nanotechnology has emerged as a multidisciplinary field of research, where scientists from different areas of specialization work together to increase their understanding of nanomaterials at the fundamental level as well as their possible applications in the industry [1-4]. Among a wide range of nanomaterials possible to manufacture now, chemically synthesized nanoparticles have their own individual importance due to their versatile applications based on their electrical, optical and magnetic properties [5-7]. Especially iron oxide nanoparticles have attracted a tremendous interest and an intensive research since the field of nanotechnology started. This happened because of iron oxides nanoparticles' novel properties particularly the type of magnetism present in them. Small iron oxide particles $(1-100 \mathrm{~nm})$ exhibit unique features that strongly differ from those of bulk counterpart [810]. These nanoparticles are of fundamental importance in many industrial applications such as a contrast agent for magnetic resonance imaging [11, 12], painting, coating, textile finishing, building materials, and in the production of ceramics as well [13]. Due to their hardness, catalytic activity, surface resistivity and other properties such as magnetic, optical and electronic they are also used as polishing agents, catalysts, gas sensors, in color imagining, in ferrofluid technology and in manufacturing of magnetic recording media which are then used in information storage devices [14, 15].

In our present work we have prepared hematite ( $\alpha$ phase of iron oxide) nanoparticles using chemical co-precipitation prolysis method. Particles after synthesis were coated using polyvinylpyrrolidone (PVP)

* corresponding author; e-mail: imran@uoregon.edu

as the coating agent. The advantage of using $\mathrm{Fe}_{2} \mathrm{O}_{3}$ nanoparticles for coating relies on their chemical stability, in contrast to the commonly used ultrasmall particles of pure metals [16]. Also compared to other phases of iron oxide (for example compared to $\gamma$-phase) $\alpha$-phase is stable for longer range of annealing times and temperatures $[17,18]$. For coating polymer we used PVP. Use of PVP for encapsulation purposes is not new (see for instance [19] where PVP is used to encapsulate Ag nanoparticles). Our reasons for choosing PVP as surfactant was based on its properties like its ability of solving into water and into other polar solvents and its easy commercial availability. In solution form, it has excellent wetting properties, too [20], which we found a necessary factor in the synthesizing process.

The aim of the studies performed in this article is first to investigate the size reduction in the hematite nanoparticles when coated with polymer PVP. This particular goal can help in manufacturing ultra-small hematite nanoparticles with superparamagnetic character which have possible applications in biomedicine (targeted drug delivery) [21-23]. Second key point of our work is to study the effect of PVP on the physical properties of $\alpha-\mathrm{Fe}_{2} \mathrm{O}_{3}$ nanoparticles (in particular the magnetic and optical properties). This study is crucial from the perspective of utilizing PVP coated $\alpha-\mathrm{Fe}_{2} \mathrm{O}_{3}$ particles in biological and chemical industry. In next sections we will exhibit our results and a detailed discussion of our findings will be presented.

\section{Experimental}

Uncoated hematite nanoparticles were obtained through the following co-precipitation prolysis method. In the first step of precipitation, appropriate amounts of ferrous chloride tetrahydrate $\left(\mathrm{FeCl}_{2} \cdot 4 \mathrm{H}_{2} \mathrm{O}\right)$ in $2 \mathrm{M} \mathrm{HCl}$ and ferric chloride hexahydrate $\left(\mathrm{FeCl}_{3} \cdot 6 \mathrm{H}_{2} \mathrm{O}\right)$ were mixed at room temperature $\left(\mathrm{Fe}^{2+} / \mathrm{Fe}^{3+}=1 / 2\right)$. Mixture was 
dropped into $200 \mathrm{ml}$ of $1.5 \mathrm{NaOH}$ solution under vigorous stirring for about $30 \mathrm{~min}$. The $\mathrm{pH}$ of the solution was constantly monitored and reactants were constantly stirred using a magnetic stirrer as the mixture was added. Next the precipitates were washed five times with deionized water to remove impurities. To separate the precipitate further from impurities the beaker contents were then centrifuged for one minute at $2000 \mathrm{rpm}$ (rotations per minute). By repeating the last step twice sample was dried at $40^{\circ} \mathrm{C}$ [24]. The acquired substance was finally grinded into a fine powder. In the second step of prolysis, the precursor was decomposed to hematite by annealing at $450^{\circ} \mathrm{C}$ for 4,5 and $6 \mathrm{~h}$ and at $650^{\circ} \mathrm{C}$ for $4 \mathrm{~h}$ in air.

PVP coated hematite nanoparticles were obtained via the microemulsion approach: polyvinylpyrrolidone solution $\left(5 \mathrm{mg}\right.$ in $50 \mathrm{ml}$ of water) was heated at $90{ }^{\circ} \mathrm{C}$ for $1 \mathrm{~h}$ with continuous agitation $(1000 \mathrm{rot} / \mathrm{min})$ to form microemulsion. Then $40 \mathrm{ml}$ of $5 \mathrm{M} \mathrm{NaOH}$ was added to the solution. Ferrite solution $(30 \mathrm{ml})$ containing stoichiometric ratio of $1: 2$ of ferrous chloride tetrahydrate $\left(\mathrm{FeCl}_{2} \cdot 4 \mathrm{H}_{2} \mathrm{O}\right)$ and ferric chloride hexahydrate $\left(\mathrm{FeCl}_{3} \cdot 6 \mathrm{H}_{2} \mathrm{O}\right)$ was added dropwise to the solution. The suspension was incubated for $1 \mathrm{~h}$ at $90^{\circ} \mathrm{C}$ with gentile stirring. Afterwards $5 \mathrm{M} \mathrm{NaOH}$ was added dropwise to this solution. The precipitate was centrifuged and washed with deionized water. The product was separated by centrifugation $(2000 \mathrm{rpm})$ and dried at $40^{\circ} \mathrm{C}$. In the prolysis step, the precursor was decomposed to PVP coated hematite by annealing in the tube furnace at $450^{\circ} \mathrm{C}$ for $4 \mathrm{~h}$ in air.

\section{Results and discussion}

\subsection{Structural characterization}

\subsubsection{XRD analysis of uncoated hematite samples}

The structure and phase purity of the samples were confirmed by performing powder X-ray diffraction (XRD) with $\mathrm{Cu} K_{\alpha}$ radiation of $\lambda=1.5405 \AA$ at room temperature. We used the XRD model JDX-11 of JEOL Company Ltd. Japan, operated at $35 \mathrm{kV}$ and $20 \mathrm{~mA}$. $\mathrm{X}$-ray diffraction pattern of uncoated hematite samples is shown in Fig. 1.

The crystallite size was calculated using Scherrer's formula $D_{h k l}=K \lambda / \beta \cos \theta$, where $D_{h k l}$ is the particle diameter in $\AA, K$ is the Scherrer coefficient taken as $0.89, \beta$ is the full width at half maximum (FWHM) and $\lambda$ is the wavelength of X-rays. It was found that samples annealed at $450{ }^{\circ} \mathrm{C}$ for $4 \mathrm{~h}(\mathrm{HU}-1), 5 \mathrm{~h}(\mathrm{HU}-2)$ and $6 \mathrm{~h}(\mathrm{HU}-$ 3 ) have some noise present but all major peaks are of pure hematite phase of iron oxide. Comparing with already reported results [25, 26], it was found that the Miller indices (hkl) corresponding to the standard peaks i.e. at $2 \theta=33.5^{\circ}, 35.6^{\circ}$, and $55.4^{\circ}$ are (104), (110), and (116), respectively, match with those for hematite. Intensity of peak at $33.5^{\circ}$ was the largest of all and if supposed to be $100 \%$ then the intensities of peaks at $35.6^{\circ}$ and $55.4^{\circ}$ were $68 \%$ and $48 \%$, respectively, which when compared with relative intensities of the peaks in the standard data [25, 26], again showed agreement. Hence even

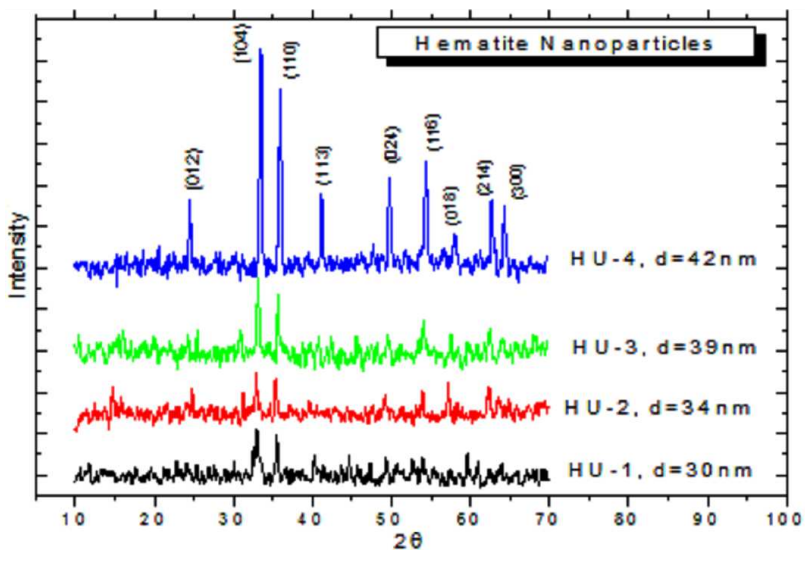

Fig. 1. XRD pattern of sample HU-1, HU-2, HU-3, and HU-4 with respective particle sizes which are calculated from three main peaks of XRD pattern (peak intensities are measured in arbitrary units).

though there is some noise present in XRD patterns, but we can safely claim in each plot (sample HU-1, HU-2, and $\mathrm{HU}-3$ ) overall phase to be of $\alpha-\mathrm{Fe}_{2} \mathrm{O}_{3}$. When sample was annealed at higher temperature (at $650{ }^{\circ} \mathrm{C}$ for $4 \mathrm{~h}$ ) we note a considerable reduction in noise as well as hematite peaks become more pronounced.

Our choice of annealing temperature and time is consistent with literature [27] where it is reported that for the preparation of impurity free hematite nanoparticles annealing at high temperatures $\left(600-700^{\circ} \mathrm{C}\right)$ is adequate.

\subsubsection{XRD analysis of coated nanoparticles}

From preparation of uncapped $\alpha-\mathrm{Fe}_{2} \mathrm{O}_{3}$ nanoparticles it was clear that for pure alpha- phase annealing at $650{ }^{\circ} \mathrm{C}$ for $4 \mathrm{~h}$ is sufficient. But keeping in view the burning point of PVP $\left(700^{\circ} \mathrm{C}\right)$ [29], annealing temperature was changed to $450^{\circ} \mathrm{C}$ for $4 \mathrm{~h}$ in air atmosphere for the coating of nanoparticles. Powder XRD (Fig. 2) confirmed the

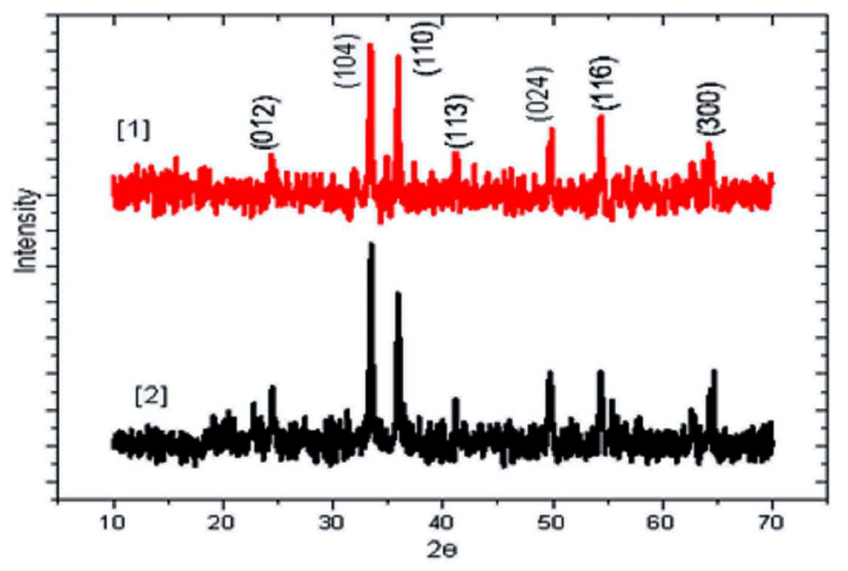

Fig. 2. Comparisons of XRDs of: [1] coated sample, $d=25 \mathrm{~nm},[2]$ uncoated sample, $d=33 \mathrm{~nm}$.

coated sample to be in hexagonal hematite phase with some noise in the XRD data. Let us note that for most of the phases of iron oxide (including hematite), this noise is 
very hard to avoid at such lower temperatures and times. Peak positions are the same for both samples. But close (enlarged) observation indicated broadening of peaks in coated sample, which corresponds to smaller size particles according to Scherrer's formula. It was observed that coating reduced particle size from $33 \mathrm{~nm}$ to $25 \mathrm{~nm}$. It was a significant decrease, indicating that PVP disallowed the hematite nanoparticles from agglomeration and experiments conducted have achieved their main goal.

\subsection{Confirmation of coating}

\subsubsection{Fourier transform infrared spectroscopy results}

The attachment of the PVP on the particle surface was further confirmed by Fourier transform infrared (FTIR) spectroscopy (where we used Fourier transform infrared spectrometer BIORAD in Excalibur series model FTS 3000MX made in USA, between the wave number $400 \mathrm{~cm}^{-1}$ to $4000 \mathrm{~cm}^{-1}$ in the percent transmittance mode). Figure $3 \mathrm{a}-\mathrm{c}$ shows the IR transmis-

TABLE I

Assignment of the absorption bands in IR spectra.

\begin{tabular}{c|c}
\hline \hline $\begin{array}{c}\text { Uncoated sample } \\
\lambda[1 / \mathrm{cm}]\end{array}$ & Assignments* \\
\hline 447 & $\mathrm{Fe}-\mathrm{O}$ lattice vibrations \\
\hline 550 & $\begin{array}{c}\mathrm{Fe}-\mathrm{O} \text { lattice vibrations } \\
\text { in uncoated hematite }\end{array}$ \\
\hline 920 & $\mathrm{C}-\mathrm{N}$ \\
\hline$*[28]$ &
\end{tabular}

TABLE II

Assignment of the absorption bands in IR spectra.

\begin{tabular}{c|c}
\hline $\begin{array}{c}\text { Pure PVP } \\
\lambda[1 / \mathrm{cm}]\end{array}$ & Assignments* \\
\hline 572 & $\mathrm{~N}-\mathrm{C}=\mathrm{O}$ bend \\
\hline 844 & $\mathrm{C}-\mathrm{C}$ ring \\
\hline 894 & $\mathrm{C}-\mathrm{C}$ ring \\
\hline 943 & $\mathrm{C}-\mathrm{C}$ ring \\
\hline 1016 & $\mathrm{C}-\mathrm{N}$ \\
\hline 1078 & $\mathrm{C}-\mathrm{N}$ \\
\hline 1224 & $\mathrm{CH}_{2}$ twist \\
\hline 1290 & $\mathrm{~N}-\mathrm{OH}$ Complex \\
\hline 1374 & $\mathrm{CH}_{2}$ bend \\
\hline 1467 & $\mathrm{C}-\mathrm{N}$ bend \\
\hline 1656 & $\mathrm{C}=\mathrm{O}$ stretching \\
\hline 2957 & asym. CH $_{2}$ ring \\
\hline 3418 & $-\mathrm{OH}$ stretching \\
\hline$*[29]$ &
\end{tabular}

sion spectra of uncoated hematite nanoparticles, pure PVP and PVP coated hematite nanoparticles, respectively. Corresponding assignments $\lambda$ of the absorption bands in the spectra are listed below in Tables I, II,
TABLE III

Assignment of the absorption bands in IR spectra.

\begin{tabular}{|c|c|}
\hline $\begin{array}{c}\text { Coated sample } \\
\lambda[1 / \mathrm{cm}]\end{array}$ & Assignments* \\
\hline 462 & Fe-O lattice vibrations \\
\hline 540 & $\begin{array}{c}\mathrm{Fe}-\mathrm{O} \text { lattice vibrations } \\
\text { in uncoated Hematite while due } \\
\text { to } \mathrm{N}-\mathrm{C}=\mathrm{O} \text { bend in pure PVP }\end{array}$ \\
\hline 838 & $\mathrm{C}-\mathrm{C}$ ring \\
\hline 935 & $\mathrm{C}-\mathrm{C}$ ring \\
\hline 1019 & $\mathrm{C}-\mathrm{N}$ \\
\hline 1074 & $\mathrm{C}-\mathrm{N}$ \\
\hline 1291 & $\mathrm{~N}-\mathrm{OH}$ Complex \\
\hline 1320 & $\mathrm{CH}_{2}$ bend \\
\hline 1453 & $\mathrm{CH}_{2}$ Scissor \\
\hline 1655 & $\mathrm{C}=\mathrm{O}$ stretching \\
\hline 2959 & Asymmetric $\mathrm{CH}_{2}$ ring \\
\hline 3379 & -OH Stretching \\
\hline
\end{tabular}

* $[28,29,30]$

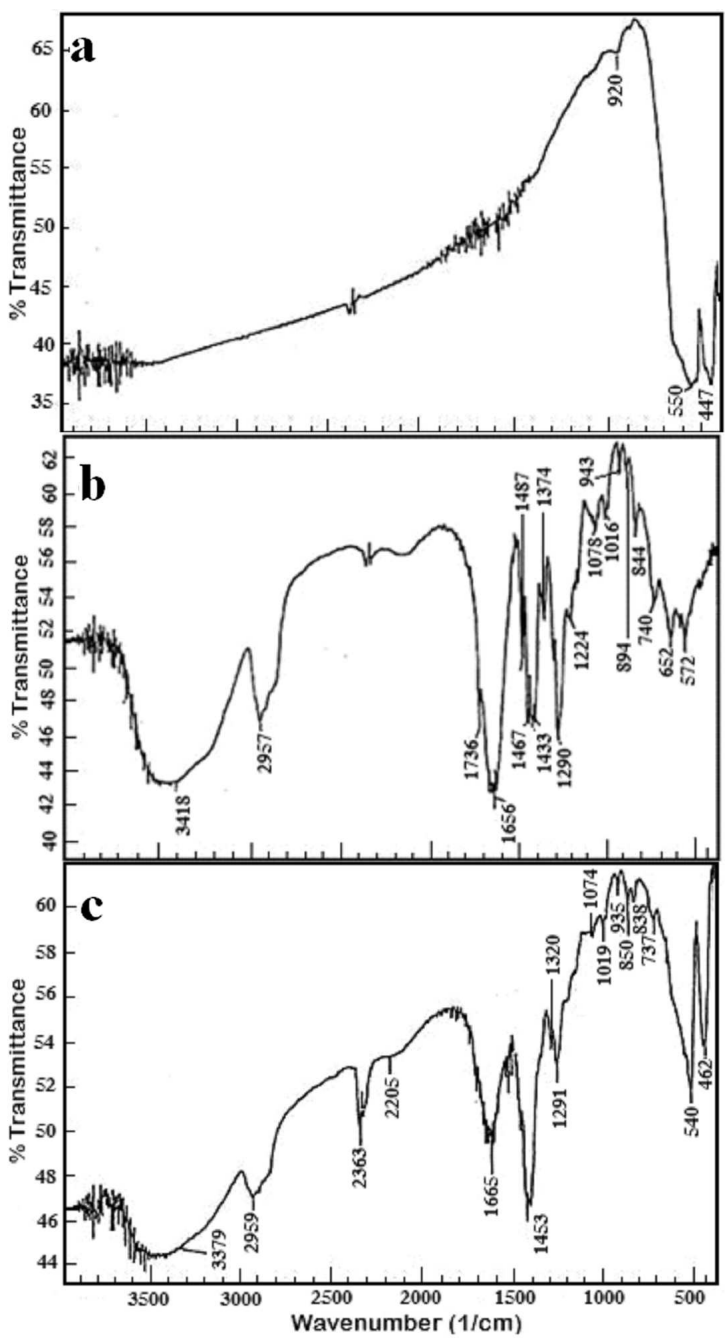

Fig. 3. FTIR graph of uncoated (a), pure PVP (b), and PVP coated (c) sample. 
and III. The spectra of the hematite nanoparticles coated with PVP exhibits absorptions corresponding to both uncoated and pure PVP samples with slight shifting in wave numbers. Absorptions at $920 \mathrm{~cm}^{-1}$ wave number in uncoated sample correspond to $\mathrm{C}-\mathrm{N}$ mode vibrations (see Table I and Fig. 3a).

This is most possibly due to the presence of some organic impurity in our uncoated sample but of course the amount of the impurity is very small as clear from the XRD analysis (Fig. 2). Dominant bands in coated sample at about 462 and $540 \mathrm{~cm}^{-1}$ are characteristics of crystalline $\alpha-\mathrm{Fe}_{2} \mathrm{O}_{3}$ [28] (see Table III and Fig. 3c), whereas the remaining absorptions can be assigned to pure PVP [29, 30]. This additive effect in absorptions is a clear indication of the presence of PVP in our coated sample.

\subsubsection{Thermogravimetric analysis curves}

The attachment of the PVP on the surface of the hematite particles was furthermore confirmed by thermogravimetric analysis (TGA) measurements (where measurements were performed on Thermogravimetric Analyzer imported from Mettler Toledo TGA/SDTA 851e DSC 823e Thermo Scientific Nicolet 6700). Analysis was performed in the presence of static air at a linear heating rate of $10^{\circ} \mathrm{C}$ from room temperature to $900^{\circ} \mathrm{C}$. TGA curves of uncoated hematite, PVP and coated hematite nanoparticles are shown in Fig. 4. It was observed that the percentage of total weight lost is larger when Hematite nanoparticles are coated with PVP $(52 \%)$ compared to that of uncoated Hematite particles (10\%). Thermogram for coated sample shows a continuous weight loss in the range of decompositions temperature of PVP (compare parts of curves for pure PVP and coated sample (Fig. 4).

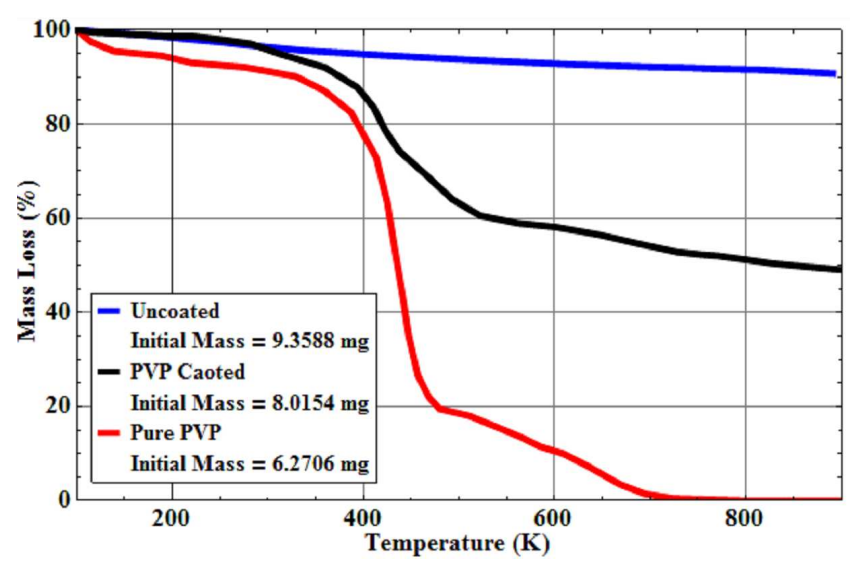

Fig. 4. TGA curves of uncoated, pure PVP, and coated sample with corresponding initial masses.

From the mass loss in percentage it is possible to make a rough estimate of the PVP content i.e. thickness of the surfactant layer on the hematite nanoparticles. Assume for simplicity that particles are spherical and monodispersed. Let us note that strictly speaking such an assumption about the shape and dispersity of nanoparticles is not valid, but we are making this assumption just to roughly estimate the dead layer thickness, which is otherwise very hard to calculate for arbitrary shapes and dispersity of particles. From TGA curve of coated nanoparticles it is quite clear that initial mass of the powder sample having say $N$ number of particles is $8.01 \mathrm{mg}$ (we shall call this mass $M_{1}$ ). We also know the average particle size from XRD for coated sample $(d=25 \mathrm{~nm})$. At about $750{ }^{\circ} \mathrm{C}$ all PVP (mass density i.e. $\rho_{1}=1.67 \mathrm{~g} / \mathrm{cc}$ ) vanishes from the sample and remaining is $N$ number of uncoated hematite particles $\left(\rho_{2}=5.07 \mathrm{~g} / \mathrm{cc}\right)$. At this temperature mass of the remaining sample is $4.7 \mathrm{mg}\left(M_{2}\right)$. Then

$$
\begin{aligned}
& M_{1}=N\left[(4 \pi / 3) \rho_{2} r_{2}^{3}+(4 \pi / 3) \rho_{1}\left(r_{1}-r_{2}\right)^{3}\right] ; \\
& M_{2}=N\left[(4 \pi / 3) \rho_{2} r_{2}^{3}\right] .
\end{aligned}
$$

As we know that coated particles have average size $25 \mathrm{~nm}$ so corresponding radius is $r_{2}=12.5 \mathrm{~nm}$, then by taking ratio of above equations and putting known constants we get $r_{1}=16.7 \mathrm{~nm}$, which means that PVP layer is roughly $4.2 \mathrm{~nm}$ thick.

\subsection{Magnetic characterizations}

We studied the magnetic properties of the prepared samples by using the vibrating sample magnetometer (VSM model BHV-50 of Riken Denishi Company Ltd., Japan). To measure the magnetic properties of the particles we filled Teflon capsule and in order to have the actual response of our samples the magnetization of the empty capsule is subtracted for each loop. The contribution of the holder turned out to be less than $1 \%$ of the samples. The masses were determined using electronic balance. Our measurements were focused on the determination of the magnetization as a function of magnetic field, temperature and its cycling (hysteresis loop, $M(H))$ and determination of various parameters from it. Loops were studied at room temperatures. Typical field range was between $-7 \mathrm{kOe}$ to $+7 \mathrm{kOe}$. $M(T)$ measurements were carried out from $100 \mathrm{~K}$ to room temperature at different fixed fields.

\subsection{1. $M-H$ loops for uncoated particles}

The $M-H$ loops for uncoated hematite samples showed weak ferromagnetic hysteresis at room temperature that is above the Morin transition temperature $T_{\mathrm{M}}$. The hematite contains both a spin canted and a defect moment. The defect moment is generally attributed to foreign cations, but it also occurs from other defects, such as the ordering vacancies in the basal plane and/or chemical impurities. Both moments can contribute to the magnetization above the Morin transition, while the spin canted moment vanishes below the Morin transition [31]. The maximum field of $7 \mathrm{kOe}$ was not enough to saturate the magnetization, so the values given are not the saturated values of magnetization.

Hysteresis loops of different uncoated samples at room temperature are shown in Fig. 5. The magnetization of the bulk sample has been reported to be approximately 
$0.75 \mathrm{emu} / \mathrm{g}$ at $10 \mathrm{kOe}[32]$. The difference in the values of magnetization could be due to the presence of "dead surface layer" [33] in our nanoparticles. So that in the core part of the hematite nanoparticles exchange interactions play the main role and promote weak ferromagnetic behavior whereas, in the outer dead surface layer, magnetic properties are modified by defects, vacancies, stresses, and asymmetrical bond environment. All these surface effects which eventually results in moments not contributing to magnetization cause this reduction in magnetization values as mentioned above.

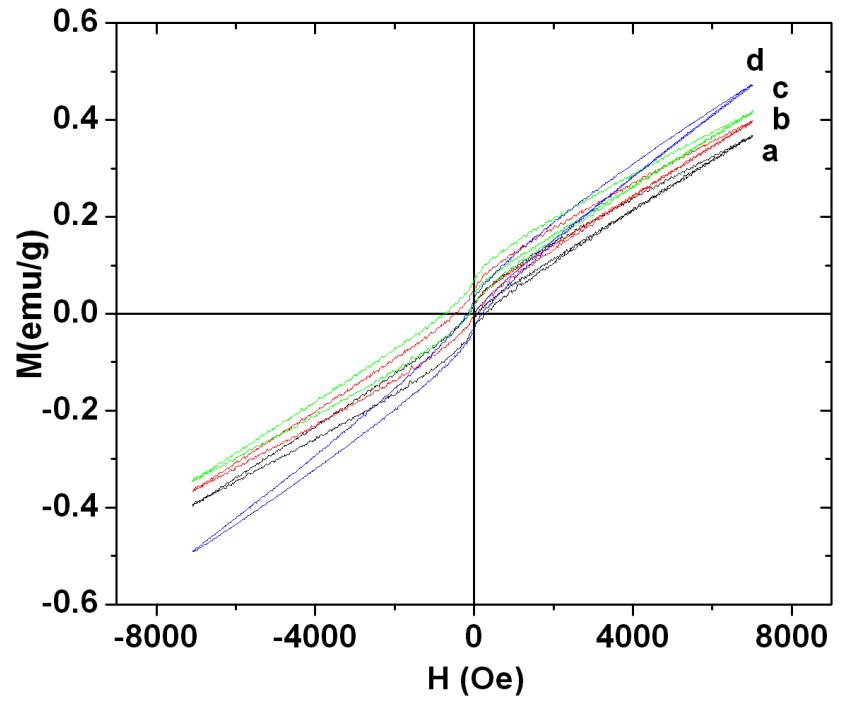

Fig. 5. Hysteresis $(M H)$ loop of uncoated samples:

(a) sample HU-1: $M_{\mathrm{r}}=0.130 \mathrm{emu} / \mathrm{g}, M_{\mathrm{s}}=0.27 \mathrm{emu} / \mathrm{g}$,

(b) sample HU-2: $M_{\mathrm{r}}=0.02 \mathrm{emu} / \mathrm{g}, M_{\mathrm{s}}=0.31 \mathrm{emu} / \mathrm{g}$,

(c) sample HU-3: $M_{\mathrm{r}}=0.03 \mathrm{emu} / \mathrm{g}, M_{\mathrm{s}}=0.33 \mathrm{emu} / \mathrm{g}$,

(d) sample HU-4: $M_{\mathrm{r}}=0.05 \mathrm{emu} / \mathrm{g}, M_{\mathrm{s}}=0.48 \mathrm{emu} / \mathrm{g}$.

Note that the thickness of the dead surface layer can be estimated through the following equation:

$$
t=(r / 2)\left\{1-\left(M_{\mathrm{S}} / M_{\mathrm{SO}}\right)^{1 / 3}\right\},
$$

where $M_{\mathrm{S}}$ and $M_{\mathrm{SO}}$ represent the saturation magnetization of the powder sample and conventional bulk sample, respectively. We find that the dead layer thickness $(t)$ is $4.5,4.4,3.9$, and $2.9 \mathrm{~nm}$ for samples HU-1, HU-2, HU-3, and HU-4, respectively. The coercivity $\left(H_{\mathrm{c}}\right)$ was found to be $337.9,162.7,148$, and 133.9 Oe for samples HU1, HU-2, HU-3, and HU-4, respectively. Also we found that, with increasing annealing time and temperature, the saturation magnetization increases. We again emphasize that, by saturation magnetization $\left(M_{\mathrm{S}}\right)$ we mean the value of magnetization achieved by our samples at the maximum applied field $H$ (which is $7 \mathrm{kOe}$ ). This may be attributed to the smaller surface/volume ratio in larger particles and hence smaller dead layer contribution. Although $c$-plane crystalline anisotropy is small but large coercivity of nanohematites is most possibly due to large magnetoelastic anisotropy caused by internal stresses. A large decrease in the coercivity with increase in the particle size is indeed a generally observed effect in many different types of magnetic nanoparticles e.g. Fe nanoparticles. It is attributed to the increasing role of the surface (for smaller sizes) and its defects in determining the anisotropy and hence the coercivity. Our results regarding coercivity are consistent with the results reported in literature [34] as well.

\subsubsection{Comparison of $M-H$ loops for uncoated and PVP coated nanohematites}

Comparison of $M-H$ loops for uncoated and coated samples at room temperature is shown in Fig. 6. We observed that coating has reduced the saturation magnetization value to a considerable amount $(0.75 \mathrm{emu} / \mathrm{g}$ to $0.5 \mathrm{emu} / \mathrm{g}$ ), while coercivity has increased from 101.2 Oe to 202.9 Oe. The suppression of the magnetization in PVP-coated samples relative to the pure $\alpha-\mathrm{Fe}_{2} \mathrm{O}_{3}$ particles is obviously due to mass effects as a result of coating of the polymer on the nanoparticles. The other possible reason is based on the fact that the polymer reduces the hematite crystallite size which resulted in the reduction of interparticle exchange interactions due to increased interparticle separations, thereby further reducing the magnetization.

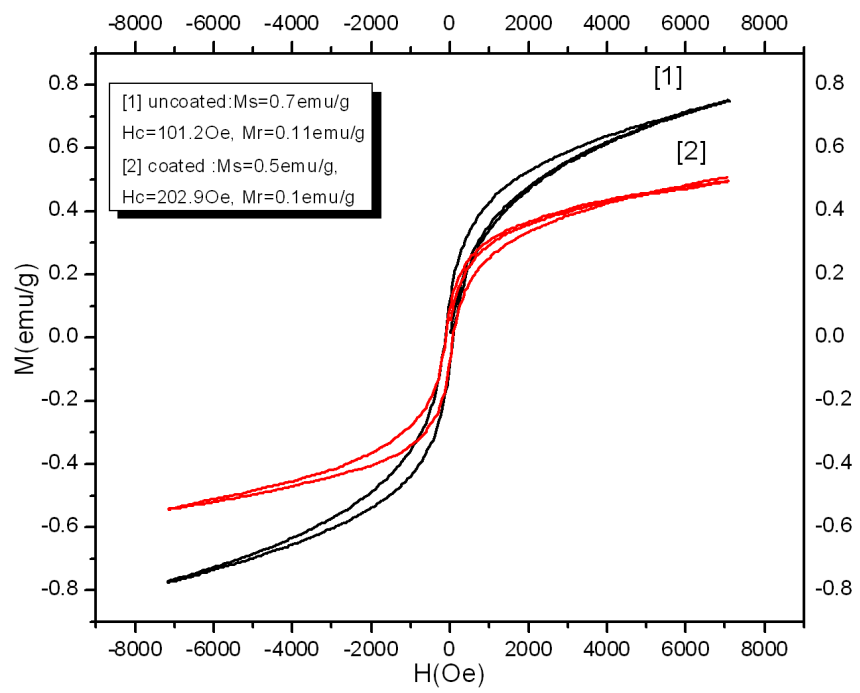

Fig. 6. Comparison of hysteresis loop for [1] uncoated hematite nanoparticles, $d=33 \mathrm{~nm}$. [2] PVP coated nanohematites, $d=25 \mathrm{~nm}$.

Increment in $H_{\mathrm{c}}$ value is remarkably unusual and needs to be explored further. At this stage we can say that this larger coercivity can be attributed to increased interparticle interaction which is working against the dipolar interactions, as it is well known that dipolar interactions try to lower $H_{\mathrm{c}}$ value.

\subsection{3. $M-T$ plots of uncoated hematite nanoparticles}

Magnetization as a function of temperature for nanohematite sample HU-4 at different applied fields is shown in Fig. 7. Data has taken at three different fields in zero field cooling (ZFC) modes. There is an abrupt change in the value of magnetization at a particular value of temperature. This change in literature is regarded as the Morin transition and corresponding temperature is called 
the Morin transition temperature $\left(T_{\mathrm{M}}\right)$ [32]. The effective Morin transition is derived from the temperature at which the magnetization has its main inflection point, half way between the antiferromagnetic state and weak ferromagnetic state values of the $M-T$ curves. This transition is an intrinsic characteristic of hematite but it depends on the hematite cluster size. At nanoscale the transition occurs at relatively lower temperatures (in between 210 and $260 \mathrm{~K}$ ) [35]. This is due to the difference of anisotropy of bulk material resulting in suppression present under different temperature conditions and hence the Morin temperature shifts to lower temperatures for smaller sized particles. The width of the Morin transition $\Delta T_{\mathrm{M}}$ is defined as the difference of the temperature at which the magnetization deviates from its smoothly varying values in the antiferromagnetic state and weak ferromagnetic state, respectively. It is quite clear that Morin transition temperature decrease as the applied field is increased. This behavior is also referred in literature [36], which is based on the fact that the high fields make hematite more ferromagnetic and less antiferromagnetic.

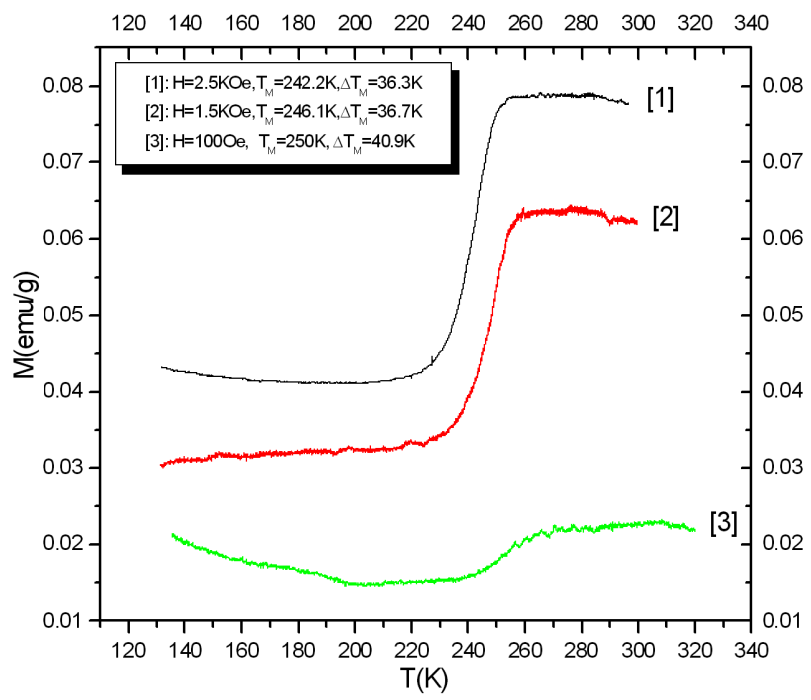

Fig. 7. Comparison of $M(T)$ for sample HU-4, $d=$ $42 \mathrm{~nm}$ at [1] $2.5 \mathrm{KOe}$ [2] $1.5 \mathrm{KOe}$, and [3] 100 Oe.

\subsubsection{Comparison of $M(T)$ curves for PVP coated and uncoated hematite nanoparticles}

The temperature dependence of $M(T)$ at 100 Oe fixed applied field for both coated and uncoated samples are reported in Fig. 8. The effective Morin transition temperature in uncoated sample is $245.4 \mathrm{~K}$, while for coated sample it reduces to $236.2 \mathrm{~K}$. Reason for this $T_{\mathrm{M}}$ decrease is the strong dependence of the Morin transition on factors like particle sizes [37], strain and defects (e.g. low crystallinity of particles, vacancies) etc. Since size of the particles after coating has reduced from $32 \mathrm{~nm}$ to $25 \mathrm{~nm}$ so this smallness in $T_{\mathrm{M}}$ is most possibly due to size reduction. Along with this it is also noted that throughout the temperature range, magnetization is less in case of coated sample as compared to its uncoated values. This is due to the fact amount of mass is more in coated sample (hematite + PVP) as compared to uncoated counterpart and measurements of magnetization are done in $\mathrm{emu} / \mathrm{g}$.

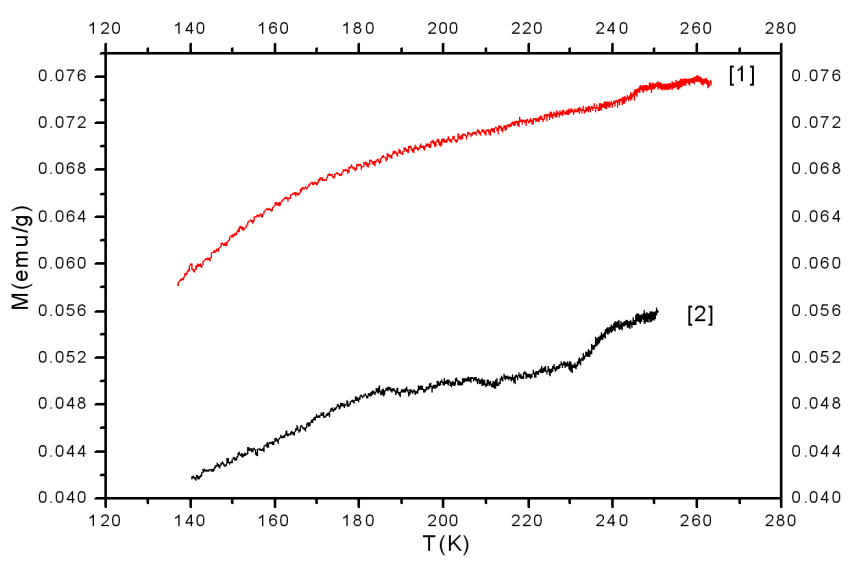

Fig. 8. Comparison of $M(T)$ for [1] uncoated sample, $T_{\mathrm{M}}=245.4 \mathrm{~K},[2]$ coated sample, $T_{\mathrm{M}}=236.2 \mathrm{~K}$ at 100 Oe.

\subsection{Optical measurements of uncoated and PVP coated nanohematites}

As despite intensive investigations, there is still controversy concerning the semiconductor properties of hematite. Hematite band gap is commonly considered to be $2.1 \mathrm{eV}$ [38] or $2.2 \mathrm{eV}$ [39], Sherman (1990) [40] has suggested that it corresponds to an $\mathrm{O}^{2-}-\mathrm{Fe}^{3+}$ charge transformation with an energy of $4.7 \mathrm{eV}$. To address this issue, in this section we present the results of optical measurements of uncoated and PVP coated hematite nanoparticles which were carried out by measuring the reflectance spectroscopy at room temperature. All spectra were taken in the range $250 \mathrm{~nm}$ to $1600 \mathrm{~nm}$ wavelengths on spectrometer model Lemda-950 Perkin Elmer. The optical band gap energy of the hematite nanoparticles were calculated from their diffuse reflectance spectra by plotting the square of the Kabulka-Munk (KM) function [41] $F(R)^{2}$ vs. energy in electron volts. The linear part of the curve was extrapolated to $F(R)^{2}=0$ to get the direct band gap energy.

\subsubsection{Optical properties of uncoated nanohematite}

Reflectance spectrum of uncoated sample annealed at $450{ }^{\circ} \mathrm{C}$ for $4 \mathrm{~h}$ is shown in Fig. 9. Direct energy band gap is calculated from the dip appearing at $536.3 \mathrm{~nm}$ (as indicated by vertical arrow in the reflectance spectrum plot of Fig. 9). KM function was then used for the measurement of band gap which turned out to be $2.1 \mathrm{eV}$ and compared well with the bulk band gap value reported in literature [22].

\subsubsection{Comparison of reflectance spectra for coated and uncoated samples}

Percentage reflectance of pure PVP, uncoated and coated sample is shown in Fig. 10. In coated sample again two dips in the spectra at $873.5 \mathrm{~nm}$ and $536.3 \mathrm{~nm}$ were 


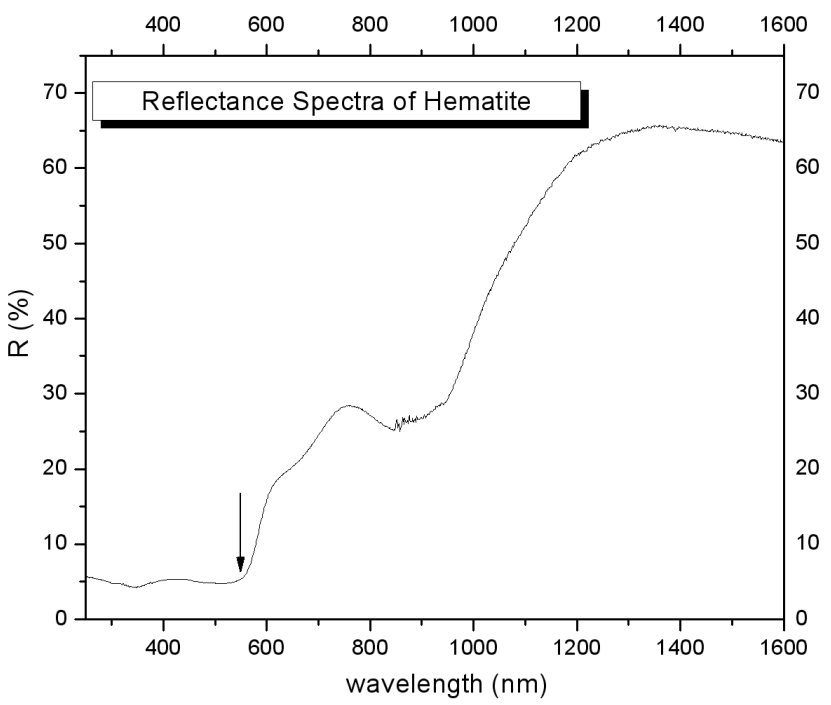

Fig. 9. Reflectance spectra of uncoated sample $(d=$ $33 \mathrm{~nm}$ ).

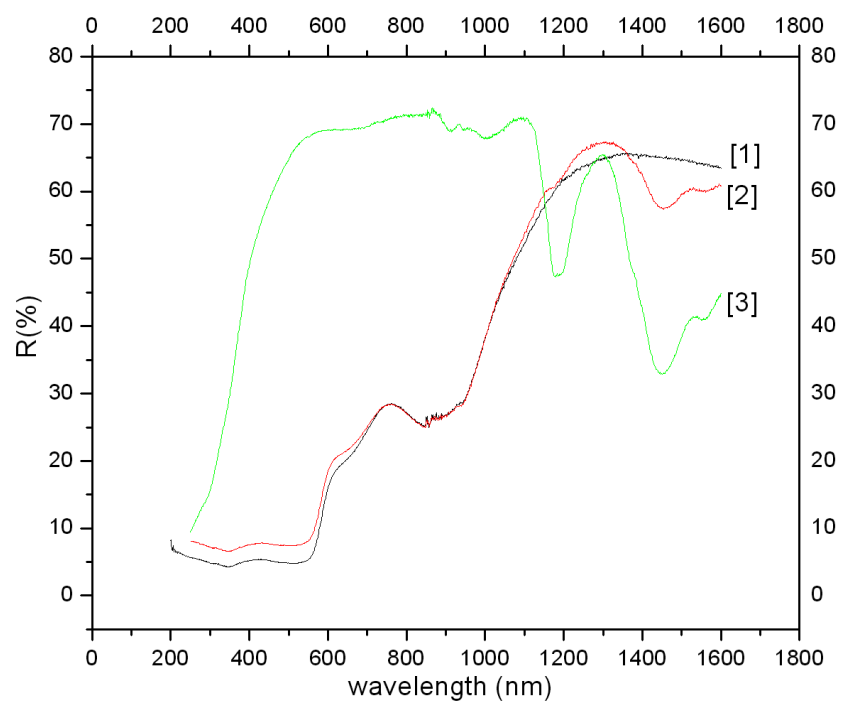

Fig. 10. Comparison of reflectance spectra of [1] uncoated, [2] coated, [3] pure PVP samples.

observed. Direct band gap was calculated from the dip appearing at $536.3 \mathrm{~nm}$. Band gap again (like uncoated case) turned out to be $2.1 \mathrm{eV}$.

As far as the band gap is concerned, there is no change observed after coating. But from the higher and lower wavelength regions of the plot it is quite clear that after coating, PVP has its reflectance signatures on the coated sample. This PVP-like behavior of the coated sample at lower and higher energy regions is another indication of the presence of the surfactant in our encapsulated sample.

\section{Summary and conclusions}

We have synthesized the alpha phase of iron oxide nanoparticles coated with PVP by microemulsion method. We found that this synthesizing technique is suitable for coating because it is relatively easier, better resulting, low cost and less time consuming. Coating was successfully confirmed by FTIR, TGA and optical UV/Vis reflectance test. We observed that surfactant disallows the agglomeration which results in smaller size particles. The particle size decreases from $33 \mathrm{~nm}$ to $25 \mathrm{~nm}$ for the same preparation conditions. Hence the experiments have succeeded in the basic goal. In magnetic studies we have observed very significant increase in the coercivity of the coated samples, as compared to uncoated. This is very remarkable considering that the coated particles are smaller in size than the uncoated, as it is well known that the coercivity of the nanoparticles increases with the volume increase. This observed increase needs to be explored further. Currently we believe it to be originating in the reduced interparticle interactions in the coated nanoparticles. Also we found that the Morin transition temperature $T_{\mathrm{M}}$ and the saturation magnetization moment decreased with decreasing size of the particles which is reflective of the basic feature of phase transition in nanoscale system which employs that the ordering temperature tends to decrease with decreasing size. The decrease of the saturation moment on the other hand, is attributed to distorted surface layer of spins that may be magnetically inert.

\section{Acknowledgments}

S.K.H. acknowledges support of Higher Education Commission Government of Pakistan for supporting this project of "Development and Study of Magnetic Nanostructure".

\section{References}

[1] C.P. Poole, F.J. Owens, Introduction to Nanotechnology, Cambridge University Press, Cambridge 2003.

[2] B. Bhushan, Springer Handbook of Nanotechnology, Springer, Berlin 2004.

[3] F. Sanchez, K. Sobolev, Constr. Build Mater. 24, 2060 (2010).

[4] K.K. Kolasinski, Surface Science: Foundations of Catalysis and Nanoscience, 3rd ed., Wiley.com (2012).

[5] V.J. Mohanraj, Y. Chen, Tropic. J. Pharmaceut. Res. 5.1, 561 (2007).

[6] G. Schmid, Nanoparticles: from Theory to Application, 2nd ed., Wiley, Weinheim 2011.

[7] S. Horikoshi, N. Serpone, Introduction to Nanoparticles, in Microwaves in Nanoparticle Synthesis: Fundamentals and Applications, Wiley-VCH Verlag, Weinheim 2013.

[8] S. Laurent, D. Forge, R.M. Port, A.C. Robic, L. Vander, R.N. Muller, Chem. Rev. 108, 2064 (2008).

[9] S.F. Hasany, N.H. Abdurahman, A.R. Sunarti, R. Jose, Curr. Nanosci. 9, 561 (2013).

[10] S.A. Teja, P. Koh, Prog. Cryst. Growth Character. Mater. 55.1, 22 (2009).

[11] S. Conroy, J.S.H. Lee, M. Zhang, Adv. Drug Deliv. Rev. 60.11, 1252 (2008). 
[12] J.S. Weinstein, C.G. Varallyay, E. Dosa, S. Gahramanov, B. Hamilton, W.D. Rooney, E.A. Neuwelt, J. Cerebral Blood Flow Metabol. 30, 15 (2009).

[13] J.M. Perez, Nature Nanotechnol. 2.9, 535 (2007).

[14] L.D. Huber, Small 1.5, 482 (2005).

[15] P.D. Stevens, J. Fan, H.M. Gardimalla, M. Yen, Y. Gao, Org. Lett. 7, 2085 (2005).

[16] K. Woo, J. Hong, S. Choi, H.W. Lee, J.P. Ahn, C.S. Kim, S.W. Lee, Chem. Mater. 16, 2814 (2004).

[17] J. Mürbe, A. Rechtenbach, J. Töpfer, Mater. Chem. Phys. 110.2, 426 (2008).

[18] X. Liu, J. Shaw, J. Jiang, J. Bloemendal, P. Hesse, T. Rolph, X. Mao, Sci. China Earth Sci. 53, 1153 (2010).

[19] H. Wang, X. Qiao, J. Chen, X. Wang, S. Ding, Mater. Chem. Phys. 94, 449 (2005).

[20] C. Leuner, D. Jennifer, Europ. J. Pharmaceut. Biopharmaceut. 50, 47 (2000).

[21] Nanotechnology in Biology and Medicine: Methods, Devices, and Applications, Ed. Tuan Vo-Dinh, Hindawi Publ. Co., 2008.

[22] Nanostructured Materials for Biomedical Applications, Eds. D.L. Shi, H.C. Gu, Hindawi Publ. Co., 2008.

[23] F. Bødker, M.F. Hansen, C. Bender Koch, K. Lefmann, S. Mørup, Phys. Rev. B 61, 6826 (2000).

[24] D. Predoi, J. Nanomater. Biostruct. 2, 169 (2007).

[25] I.V. Chernyshova, M.F. Hochella Jr., A.S. Madden, Phys. Chem. 9, 1736 (2007).

[26] R. Zboril, M. Mashian, D. Krausova, in: Mossbauer Spectroscopy in Material Sciences, Eds. M. Migleierine, D. Petridis, Kluwer Academic, Dordrecht 1999, p. 49.
[27] G. Cao, , Imperial College Press, London 2004.

[28] X. Wang, L. Gao, H. Zheng, M. Ji, T. Shen, Z. Zhang, J. Cryst. Growth 269, 489 (2004).

[29] N. Sahiner, N. Pekel, O. Guven, React. Funct. Polym. 39, 139 (1999).

[30] A. Alquadarni, S. Annapoorni, S. Larnba, Eur. Phys. J. B 39, 19 (2004).

[31] R. Skomsky, J.M.D. Coey, Permanent Magnetism, Institute of Physics Publ., 1999.

[32] X. Batlle, N. Pérez, P. Guardia, O. Iglesias, A. Labarta, F. Bartolomé, L.M. García, J. Bartolomé, A.G. Roca, M.P. Morales, C.J. Serna, J. Appl. Phys. 109, 07B524 (2011).

[33] T.P. Raming, A.J.A. Winnubst, C.M. van Kats, A.P. Phillips, J. Coll. Interface Sci. 249, 346 (2002).

[34] R.H. Kodama, J. Magn. Magn. Mater. 200, 359 (1999).

[35] L. Neel, Ann. Phys. (Paris) 249, 4 (1949).

[36] C.C. Shull, W.A. Strauser, F.O. Wollan, Phys. Rev. B 1383, 333 (1951).

[37] M.Z. Dang, D.C. Rancourt, J.E. Dutrizac, G. Lamarche, R. Provencher, Hyperf. Interact. 117, 271 (1998).

[38] B. Gilbert, C. Frandsen, E.R. Maxey, D.M. Sherman, Phys. Rev. B 79, 035108 (2009).

[39] D.M. Sherman, in: Spectroscopic Characterization of Minerals and Their Surfaces, American Chemical Society, Washington (DC), 1990, p. 284.

[40] D.A. Wheeler, G. Wang, Y. Ling, Y. Li, J.Z. Zhang, Energy Environm. Sci. 5, 6682 (2012).

[41] R.M. Cornell, U. Schertmann, Iron Oxides: Properties and Characterization, VCH Publ., Weinheim 1991. 